\title{
An Experimental Investigation on the Wind-driven Runback Motion of Water Droplets over Solid Surfaces with Different Wettabilities
}

\author{
Liqun Ma, Zichen Zhang, Hui Hu* \\ Department of Aerospace Engineering, Iowa State University, Ames, Iowa 50014, USA \\ *Corresponding authors: huhui@iastate.edu
}

\begin{abstract}
Extend Abstract
Aircraft icing is widely recognized as one of the most serious weather hazards to flight safety. Specially designed hydro-/ice-phobic coatings are currently undergoing development for aircraft icing mitigation. It was found that hydro-/icephobic coatings would delay the ice accretion iover airframe surfaces so that the impacted supercooled water droplets could be blown away by the airflow from the airframe surface before being frozen into ice. It is of fundamental importance to understand the wind-driven runback behavior of water droplets over surfaces treated with different coatings, since the corresponding knowledge would be very helpful and essential to develop more efficient anti-/de-icing systems for aircraft icing protection.

With the rapid development of surface engineering, a series of specially designed surface coatings succeed in icing mitigation using airflow to remove the remained water. While various hydro-/ice-phobic coatings/surfaces have been developed in recent years, the "state-of-the-art" icephobic coatings/surfaces can be generally divided into three categories, i.e., 1). Lotus-leaf-inspired superhydrophobic surfaces (SHS) with micro-/nano-scale surface textures to achieve very high contact angles (typically $>150^{\circ}$ ); 2 ). Pitcherplant-inspired slippery liquid infused porous surfaces (SLIPS) with a layer of liquid lubricant (which is immiscible with water) being sandwiched between ice and solid substrate materials; and 3). Icephobic elastic materials/surfaces with deformable structures/surfaces. SHS has a water droplet contact angle (CA) larger than $150^{\circ}$ and a sliding angle (SA) less than $10^{\circ}$. SHS always has a hierarchical structure which is similar to the lotus leaf, and water droplets on SHS appear as water beads which can easily roll off the surface by wind or gravity before frozen. Another strategy to reduce ice adhesion strength to a solid surface is to use a layer of liquid lubricant, which is immiscible with water, between ice and the solid surface. The use of such lubricated surfaces was investigated as early as 1960s, and has gained increasing attentions again recently with the introduction of a concept called Slippery Liquid-Infused Porous Surfaces (SLIPS). SLIPS concept is inspired by the Nepenthes pitcher plants, which have evolved highly slippery, liquidinfused micro-textured rim to capture insects. SLIPS surfaces were not only found to be able to suppress ice/frost accretion by effectively removing condensed moisture even in high humidity conditions, but also exhibit at least an order of magnitude lower ice adhesion than most SHS coatings. More recently, elastic materials/surfaces, such as Polydimethylsiloxane or PDMS in short, which would be structurally deformed/altered dynamically upon applying extra mechanical stress, have also been suggested for icing mitigation. Elastic materials display ultra-low adhesion to ice due to their low work of adhesion and liquidlike deformability, while maintaining good mechanical durability due to their solid-like rigidity. It is found that water droplets would not only be more readily rebounding away from the surface after impingement, but also be able to roll away before frozen due to the hydrophobicity of PDMS. Considering the differences in wettabilities and mechanisms of water repellency, it is necessary to have a systematic understanding of how efficient the surfaces are when the aerodynamic force is applied to remove the adhered water droplets.
\end{abstract}


In the present study, a comprehensive experimental campaign was conducted to characterize the transient runback behaviors of wind-driven water droplets over the surfaces of test plates coated with different hydro-/icephobic coatings (i.e., SHS, SLIPS and PDMS). A high-resolution Particle Image Velocimetry (PIV) system was used to achieve quantitative measurements of the velocity field of the airflow around the wind-driven water droplets on the test surfaces with different wettabilities. With the detailed PIV measurements of the airflow field around the runback water droplets and the droplet profiles, the aerodynamic forces and the adhesion forces acting on the water droplets were estimated. While Fig. 1 shows the experimental setup used in the present study, Fig. 2 to Fig. 3 given some of the typical measurement results. More measurement results and comprehensive analysis and discussions will be provided in the full version of this research paper.

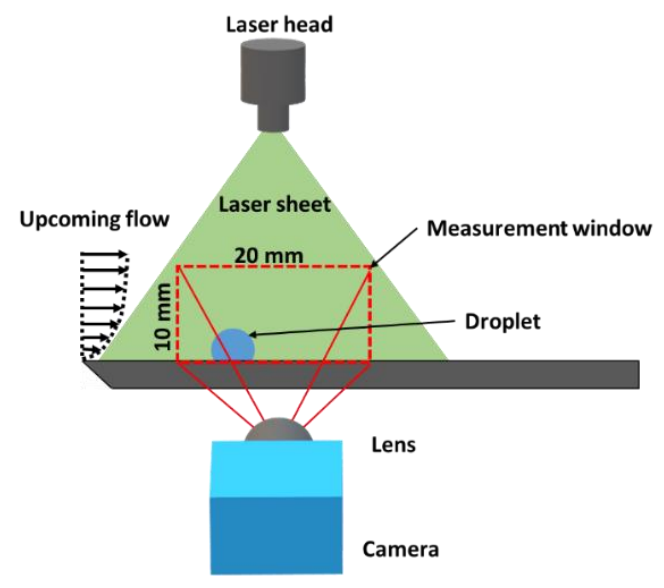

Fig. 1: Experimental setup of PIV measurements to characterize wind-driven runback of water droplets.

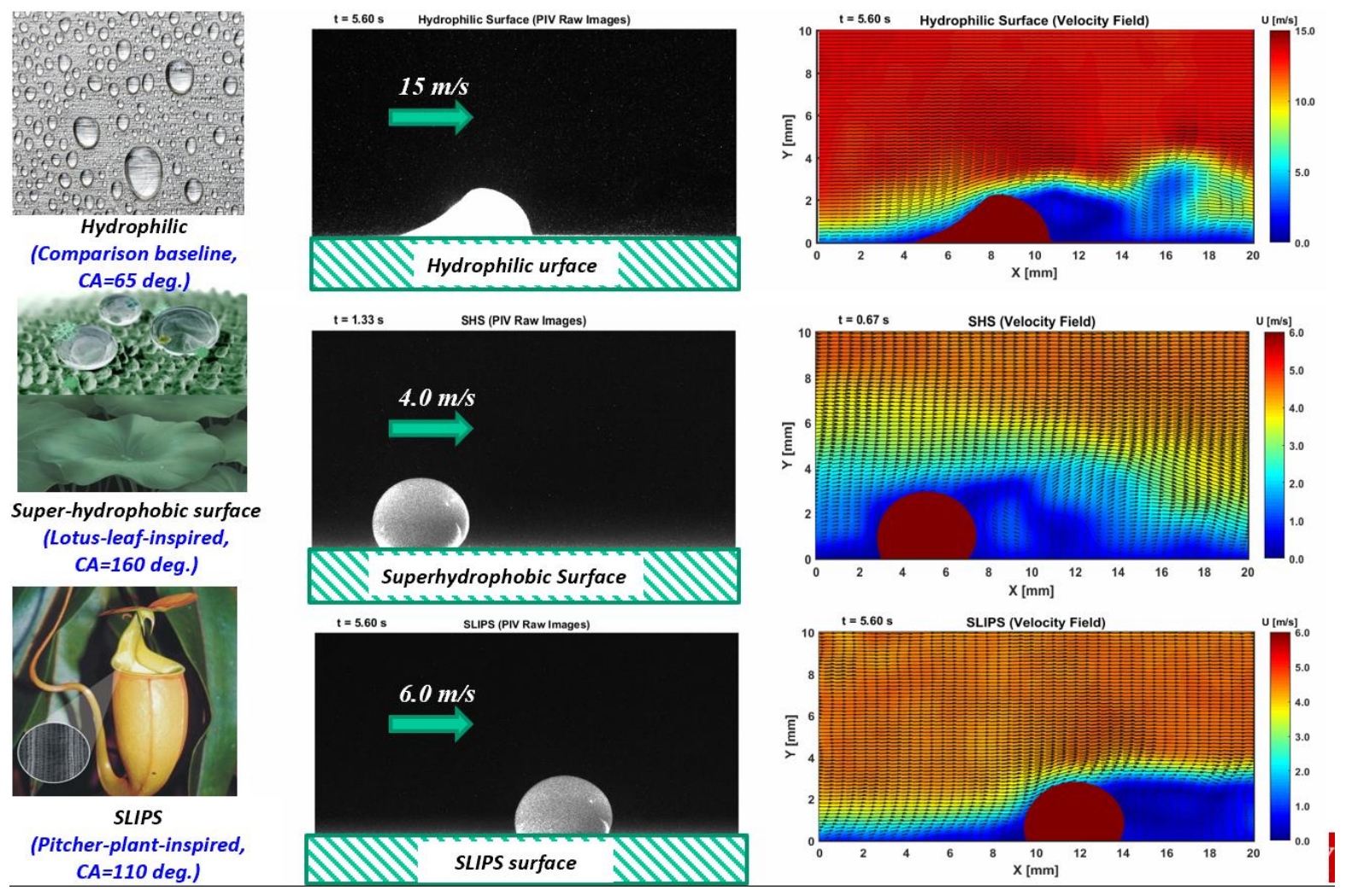

Fig. 2. PIV measurements of wind-driven water droplets over test plates coated with different coatings. 